\title{
Risk Factors for Colonization with Yeast Species in a Veterans Affairs Long-term Care Facility
}

\author{
Sara A. Hedderwick, MRCP," Jim Y. Wan, PHD, ${ }^{\dagger}$ Suzanne F. Bradley, MD," \\ Jon A. Sangeorzan, MD," Margaret S. Terpenning, MD, and Carol A. Kauffman, MD"
}

OBJECTIVES: To assess colonization and serious infection with yeasts and the risk factors that are associated with colonization by these organisms.

DESIGN: Monthly surveillance for colonization and infection over a period of 2 years.

SETTING: A long-term-care facility (LTCF) attached to an acute-care Veterans Affairs Medical Center.

PARTICIPANTS: The 543 men and 10 women in the facility. MEASUREMENTS: Colonization and serious infection rates with yeasts. Analysis of risk factors associated with yeast colonization of residents.

RESULTS: Colonization rates were relatively stable during the 2 -year period ( $53 \pm 1.8 \%$ patients colonized per month). Candida albicans was the most common colonizer, found in $35 \pm .9 \%$ of patients colonized per month. The pharynx was the most commonly colonized site, with $41 \pm 1.4 \%$ of patients per month with pharyngeal colonization. Eightyfour percent of patients remaining in the facility for 3 or more months were colonized by yeast at some time during their stay. Presence of neurogenic bladder, leg amputation, or a low serum albumin were independently associated with yeast colonization; neither diabetes mellitus nor functional status was a risk factor for colonization by yeasts. Only four serious yeast infections in four patients (esophagitis and three urinary tract infections) were found during the 2-year period; all infections occurred in patients who were colonized by yeasts previously.

CONCLUSION: In our LTCF, colonization of patients by yeasts occurred commonly in those residents remaining in the facility for 3 or more months. However, serious yeast infections occurred infrequently. It is likely that colonization of residents of LTCFs by yeasts may only become clinically important when the patient is transferred to an acute-care hospital where additional risk factors may allow the development of serious yeast infection. J Am Geriatr Soc 46:849-853, 1998.

From the "Department of Veterans Affairs Medical Center, University of Michigan Medical School, Ann Arbor, Michigan, and tUniversity of Tennessee, Memphis, Tennessec.

Address correspondence and reprint requests to Carol A. Kauffman, MD, Veterans Affairs Medical Center, 2215 Fuller Rd., Ann Arbor, MI 48105.

\begin{abstract}
Candida spp have emerged as significant nosocomial $C_{\text {pathogens in the acutc-care hospital during the past }}$ decade; indeed, they are now the fourth leading cause of bloodstream infections and the sixth most common nosocomial pathogen in the United States. ${ }^{1,2}$ The epidemiology of Candida infections is still controversial. Infection is believed to result from invasion from the patient's own endogenous colonizing flora, ${ }^{3-7}$ although increasing reports of outbreaks of Candida infections support exogenous acquisition of Candida spp as important in some cases. ${ }^{8-12}$ Thus, the clinical importance of Candida colonization at various body sites is debated. Reagen et al. showed that in 15 of 16 patients with various malignancies, the previous colonizing and bloodstream isolates of Candida species were identical. ${ }^{4}$ Other authors have shown a similar temporal relationship between colonization and subsequent candidemia with the same strain of Candida. ${ }^{3}$ In addition, a retrospective, case-control study of hospitalized patients showed that colonization was a risk factor for subsequent candidemia. ${ }^{5}$
\end{abstract}

\section{For editorial comment, see $\mathrm{p} 916$}

There are few data regarding the epidemiology of Candida colonization of residents of long-term care facilities (LTCF). Jackson et al. reported that $4 \%$ of all infections within the LTCF population were attributable to Candida spp, with an incidence of 0.28 per 1000 resident days. ${ }^{13}$ For other microbial pathogens, such as methicillin-resistant Staphylococcus aureus (MRSA) and vancomycin-resistant enterococci, trends seen in large acute-care hospitals are mirrored in LTCFs within a few years. ${ }^{14,15}$ Thus, yeast colonization of residents in a LTCF may predispose to infection. This report provides estimates of the rates and predominant Candida spp responsible for yeast colonization in residents of a LTCF associated with a Veterans Affairs hospital and identifies putative risk factors associated with yeast colonization in this population.

\section{MATERIALS AND METHODS}

\section{Facility and Patients}

Patients were residents of the Ann Arbor Veterans Affairs Nursing Home Unit, a 120 -bed LTCF attached to a 300-bed acute-care Veterans hospital. Patients were admitted to the facility for long-term care, rehabilitation, or geriatric evaluation. From June 1, 1989, until May 31, 1991, all patients who were resident initially or admitted subsequently were 
studied for colonization with various yeast-like organisms. Patients requiring intravenous therapy were excluded.

On admission to the study, the following information was recorded for all patients: age, sex, underlying chronic diseases, previous hospitalization, and nutritional status. Functional status was evaluated as follows: Group I patients were ambulatory, requiring minimal assistance in activities of daily living; Group II patients required minor assistance in activities of daily living and had intermittent bladder incontinence; Group III patients required assistance for almost all activities of daily living and had incontinence of bowel and/or bladder. Cultures of body sites were done at admission, and monthly thereafter. If the patient was transferred to the acute-care facility, additional cultures were performed when the patient returned to the LTCF.

\section{Study Design}

The study of colonization with yeasts was part of a project that followed patients in a LTCF to assess colonization with several different organisms, including MRSA, highlevel aminoglycoside-resistant enterococci, ceftriaxone- and aminoglycoside-resistant Gram-negative bacilli, and yeasts. ${ }^{14,16-18}$

\section{Definitions}

Colonization was defined as one or more positive cultures for yeasts from any site. For patients followed for at least 3 months, the definitions applied were: persistent colonization was defined as having a culture positive for yeast in at least one site on every occasion that cultures were performed; probably persistent colonization was defined as a ratio of positive to total cultures performed of between 0.8 and 1 ; intermittent colonization was believed to have occurred when patients had more than one culture positive for yeast but were not persistently or probably persistently colonized; transient colonization was defined as only one culture positive for yeast. Infections with yeast were defined using Centers for Disease Control and Prevention criteria. ${ }^{19}$

\section{MicrobiologicalMethods}

Colonization for yeasts was assessed in the oropharynx, perineum, rectum, and wounds. Sampling was accomplished using sterile rayon applicator sticks, which were placed immediately in Stuart transport media. and, within 1 hour, were streaked onto Sabouraud dextrose agar (Difco Inc., Detroit, MI) containing $50 \mu \mathrm{g} / \mathrm{mL}$ gentamicin sulfate (Schering Inc., Summit, NJ) using a semiquantitative, four-quadrant method of streaking. Plates were incubated at room temperature for 72 hours, the amount of growth on the plates was quantified as $1+$ to $4+$, and colonies shown to be yeasts by direct microscopy were isolated.

Germ tube formation for identification of Candida albicans was assessed by incubating a small inoculum of organisms in $0.5 \mathrm{~mL}$ of fetal bovine serum at $35^{\circ} \mathrm{C}$ for 2 hours and then viewing the preparation under direct microscopy. Yeasts that were germ tube negative were speciated by the API Yeast 20C System (Sherwood Medical Products, Plainview, NY).

\section{Statistical Methods}

Data for risk factor analysis were analyzed with the use of SAS. ${ }^{20}$ Categorical variables were analyzed by univariate analysis with the Chi-square or Fisher's exact test; continuous variables were analyzed with the Student's $t$ test or
Mann-Whitney rank sum test. A $P$ value of $<.05$ was considered significant on two-tailed testing. Multivariate analysis was done by stepwise logistic regression, and the limit for entering and removing variables was .05 .

\section{RESULTS}

Five hundred fifty-three patients were entered into the study during the 2-year period and were followed for a mean of $4.3 \pm 1.0$ months. The mean age of the 543 men and 10 women was $64 \pm .5$ years.

\section{Rate of Colonization with Yeasts}

Colonization by yeasts at any site was relatively constant during the 2-year period. The mean monthly colonization rate was $53 \pm 1.8 \%$. Regardless of site, the most common organism found to be colonizing patients was C. albicans $(35 \pm .9 \%$ ) (Table 1$)$. The pharynx and rectum were the most frequently colonized areas (Figure 1). Among colonized patients, yeasts were isolated simultaneously from one, two, three, or four sites in $63 \%, 27 \%, 9 \%$, and $1 \%$ of occasions, respectively. Of those patients who were colonized with yeasts at any site, $78 \%$ had only one species present on each occasion, whereas $20 \%$ and $2 \%$ had two or three or more species isolated, respectively.

\section{Acquisition and Persistence of Yeast Colonization}

Of the 286 patients who had cultures performed on three or more occasions, 170 patients $(59 \%)$ were colonized with yeast at entry to the study; 71 patients $(25 \%)$ acquired yeast after their entry into the study (first culture negative but at least one subsequent culture positive for yeast); and yeasts were never isolated from 45 patients (16\%) (Table 2). Yeast species were persistently or probably persistently isolated in 75 patients $(26 \%)$, whereas $120(42 \%)$ and $46(16 \%)$ of patients had intermittent and transient yeast colonization, respectively. C. albicans and C. glabrata were the species most commonly isolated from all patients, regardless of their colonization status. Persistence was associated with pharyngeal more than with rectal colonization; $93 \%$ of persistently colonized patients had Candida in their pharynx, but only $64 \%$ of intermittently and transiently colonized patients had pharyngeal colonization.

\section{Risk Factors for Colonization with Yeast Species}

Evaluation of risk factors present on admission to the LTCF revealed that seven variables were associated with

\begin{tabular}{lc}
\hline \multicolumn{1}{c}{ Table 1. Yeast Colonization Rates per Month by Species } \\
\hline Yeast Species & $\begin{array}{c}\text { Mean Percent } \pm \text { SEM, } \\
\text { Patients Colonized/Month }\end{array}$ \\
\hline C. albicans & $35 \pm 0.9$ \\
C. glabrata & $17 \pm 1.9$ \\
C. parapsilosis & $4 \pm 0.1$ \\
C. tropicalis & $2.5 \pm 0.6$ \\
C. pseudotropicalis & $1.5 \pm 0.3$ \\
Other species* $^{*}$ & $6 \pm 0.4$
\end{tabular}

* Includes S. cerevisiae, T. beigelii, C. lusitaniae, C. stellatoidea, C. guilliermondii, C. lambica, B. capitatum, C. lipolytica, R. rubra, Pr. wickerhamii, C. kefyr, R. glatinis, H. anomala, Pr. stagnora. 


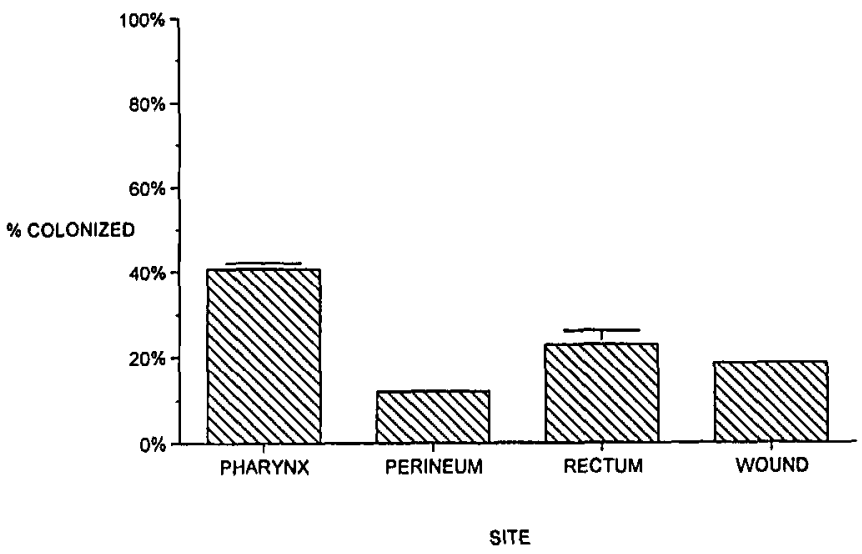

Figure 1. Monthly colonization rate (mean \pm SEM) by yeasts in residents of a long-term care facility. Percentage of wound colonization is expressed as a proportion of residents with wounds that were cultured.

yeast colonization at any site (Table 3 ). However, using stepwise logistic regression, only three of these, namely the presence of a neurogenic bladder, leg amputation, or a low serum albumin concentration, were associated independently with yeast colonization (Table 4).

When only those patients with three or more cultures performed were considered, there were six variables associated with persistent or probably persistent colonization with yeasts (Table 5). Factors that remained independently associated with persistent/probably persistent yeast colonization were previous tuberculosis, depression, the presence of wounds, and an indwelling urinary catheter (Table 6). Previous antibiotic therapy, diabetes mellitus and functional status were not associated with either persistent/probable persistent colonization or with overall colonization.

\section{Infections Caused by Yeasts}

During the 2-year study period, four infections attributable to yeasts were identified in four different patients. Three of these were urinary tract infections (UTI), and one was Candida esophagitis diagnosed by endoscopy in a patient undergoing radiotherapy for an astrocytoma. Two of the three UTIs were caused by C. albicans in patients who were

Table 2. Acquisition and Persistence of Yeast Colonization in Patients Who Had Cultures Performed on Three or More Occasions $(N=286)$

\begin{tabular}{lr}
\hline \multicolumn{1}{c}{ Colonization Status } & $\begin{array}{c}\text { Number } \\
\text { (\%) of } \\
\text { Patients }\end{array}$ \\
\hline Never colonized & $45(16)$ \\
Colonized & $241(84)$ \\
Transient colonization & $46(16)$ \\
Intermittent colonization & $120(42)$ \\
Probably persistent colonization & $29(10)$ \\
Persistent colonization & $46(16)$ \\
Colonized at entry to nursing home & $170(59)$ \\
Colonization acquired in nursing home & $71(25)$ \\
\hline
\end{tabular}

Table 3. Univariate Analysis of Factors Associated with Colonization by Yeasts $(\mathrm{N}=553)$

\begin{tabular}{lccc}
\hline & \multicolumn{2}{c}{ Percent of Patients } \\
\cline { 2 - 3 } Factor & Colonized & $\begin{array}{c}\text { Not } \\
\text { Colonized }\end{array}$ & $P$ Value \\
\hline Underlying conditions & & & \\
Recurrent UTIs & 15 & 9 & .033 \\
Neurogenic bladder & 15 & 7 & .015 \\
Presence of wounds & 38 & 27 & .014 \\
Leg amputation & 13 & 6 & .018 \\
$\quad$ Dementia & 21 & 13 & .028 \\
Devices & & & .047 \\
Gastrostomy tube & 4 & 1 & .009 \\
Nutritional parameters & & & \\
$\quad$ Serum albumin & $3.7 \pm 0.6$ & $3.9 \pm 0.6$ & .009 \\
$\quad$ (mean \pm S.D.) & & & \\
\hline
\end{tabular}

previously colonized by $C$. albicans. The third UTI was caused by a germ-tube negative yeast (not speciated further) in a patient previously colonized by C. albicans in the rectum and $C$. tropicalis in the oropharynx. The patient who subsequently developed esophagitis was colonized with C. albicans in the oropharynx and rectum. Thus, all yeast infections occurred in patients who were identified as previously colonized with yeasts.

\section{DISCUSSION}

The incidence of nosocomial yeast infections has increased dramatically during the past decade because of increased numbers of immunocompromised patients, the use of immunosuppressive drug regimens and broad-spectrum antibiotics, and the more aggressive use of life-support systems in the intensive care unit. ${ }^{5-7}$ Disseminated Candida infections are well documented in burn patients, low birth-weight infants, recipients of total parenteral nutrition, patients receiving hemodialysis, and those with intravascular catheters in place. ${ }^{4,5,8,12}$

Surprisingly little is known regarding yeast colonization and infection in patients in LTCFs. Jackson et al. reported that $4 \%$ of all infections in LTCF residents followed for a 3 -year period were attributable to Candida spp. ${ }^{13}$ These were all mucocutaneous infections, and no serious infections caused by yeasts were reported. Similarly, vaginal Candida infections were found in $7 / 186(4 \%)$ of patients in a community LTCF. ${ }^{21}$ In another study assessing only oropharyngeal

Table 4. Factors Associated Independently with Colonization by Yeasts $(\mathrm{N}=553)$

\begin{tabular}{lllc}
\hline \multicolumn{1}{c}{ Factor } & $\begin{array}{c}95 \% \\
\text { Odds } \\
\text { Ratio }\end{array}$ & $\begin{array}{c}\text { Confidence } \\
\text { Interval }\end{array}$ & P Value \\
\hline Neurogenic bladder & 2.1 & $1.06-4.0$ & 0.032 \\
Leg amputation & 3.0 & $1.37-6.48$ & 0.006 \\
Serum albumin & 0.67 & $0.49-0.92$ & 0.014 \\
\hline
\end{tabular}


Table 5. Univariate Analysis of Factors Associated with Persistent/Probably Persistent Colonization by Ycasts of Patients with Threc or More Cultures Performed $(\mathrm{N}=286$ )

Percent of Patients

\begin{tabular}{lc}
\hline & Not \\
Persistently/Probably & Persistently/Probably \\
Persistently Colonized & Persistently Colonized
\end{tabular}

$P$ Value

Factor

Persistently Colonized

Persistently/Probably
Persistently Colonized

Underlying conditions

Depression 32

Prior tuberculosis 15

Presence of wounds 53

9016

Devices

Urinary catheter

Prior intravenous catheter

5

34

.019

.003

Nutritional parameters

Serum albumin (mean $\pm S D$ )

$3.6 \pm 0.6$

1

.022

$\begin{array}{lll}4 & 0 & .025\end{array}$

Table 6. Factors Associated Independently with Persistent/ Probably Persistent Colonization by Yeasts of Patients with Three or More Cultures Performed ( $N=286$ )

\begin{tabular}{lcccc}
\hline \multicolumn{1}{c}{ Factor } & $\begin{array}{c}\text { Odds } \\
\text { Ratio }\end{array}$ & $\begin{array}{c}95 \% \\
\text { Confidence } \\
\text { Interval }\end{array}$ & $P$ Value \\
\hline Tuberculosis & 4 & $1.61-9.75$ & .003 \\
Depression & 2.4 & $1.23-4.50$ & .01 \\
Presence of wounds & 2.7 & $1.54-4.91$ & $<.001$ \\
Urinary catheter & 17.3 & $1.68-178.6$ & .017 \\
\hline
\end{tabular}

candidiasis, $38 \%$ of denture-wearing residents of a LTCF had chronic atrophic candidiasis. ${ }^{22}$

Few yeast infections occurred during the 2-year period of the study. One drawback of our study was that it was not designed to detect mucocutaneous yeast infections so that vaginitis and oral candidiasis probably occurred but were not documented. However, all serious infections, such as bacteremia, esophagitis, and urinary tract infection, were documented and were found to be uncommon. Only four such infections occurred, and all four occurred in previously colonized patients. Three of these four infections were urinary tract infections.

Our study did not assess funguria per se, and presumably this was higher than actual Candida urinary tract infection. Overall, however, the number of urinary tract infections appeared lower than that noted by others. ${ }^{23}$ This could be related to the low proportion of women in our study and the infrequent use of chronic indwelling catheters. Both of these factors seem to carry an increased risk of fungal urinary tract infections. ${ }^{23-25}$ In a previous study, we noted that Candida species were the third most common colonizing organism in patients who underwent intermittent urethral catheterization, but fungal urinary tract infection was not found in that population. ${ }^{26}$ Thus, our predominantly male population and our policy of rarely using chronic indwelling catheters may have contributed to the low rate of urinary tract infections.

The pathogenesis of yeast infection is believed to bc caused by invasion by the patient's own colonizing flora. ${ }^{3-7}$ In acute-care hospitals, previous colonization of patients by yeasts has been shown to be associated with candidemia, and a temporal trend between yeast colonization and subsequent serious Candida infection has been demonstrated..$^{3-5}$ No such association has been shown in LTCF residents, but the low number of serious Candida infections that occur within LTCF may make such an association difficult to demonstrate.

Yeast colonization has been studied in various patient populations, including those with HIV infection, diabetes mellitus, adults and neonates in the intensive care setting, and patients in acute-care hospitals. ${ }^{27-32}$ Such studies have shown that colonization with Candida spp varies little with age. ${ }^{28,33}$ Oral Candida colonization in the absence of clinical disease was found to occur in $59 \%$ of healthy community-dwelling individuals aged 60 years or older, and yeasts were found in saliva and urine in $49 \%$ and $11 \%$, respectively, of asymptomatic patients over 45 years. ${ }^{28,33}$ These rates did not vary significantly between age groups. Similarly, the overall rate of colonization with yeasts in the patients that we studied was $53 \%$. C. albicans and C. glabrata were the yeasts isolated most commonly, in accordance with studies of other patient populations. ${ }^{28,34,35}$

Patients who remained in the facility for 3 or more months were more likely to be colonized with yeasts ( $84 \%$ of patients were colonized); $59 \%$ of these patients were colonized before they entered the LTCF, and $25 \%$ acquired yeast while they were there. Colonization was mostly intermittent $(42 \%)$, but more persistent colonization with yeasts occurred in $26 \%$ of patients. Only $16 \%$ of patients remained free of yeast colonization during their stay.

Risk factors that were associated with yeast colonization by logistic regression analysis included the presence of a neurogenic bladder, leg amputation, and a low serum albumin. Surprisingly, previous antibiotic therapy, poor functional status, and diabetes mellitus were not associated with yeast colonization. Others have noted no significant differences in the yeast colonization rates found in diabetic compared with non-diabetic patients. ${ }^{21,22,28}$ Neither salivary nor urinary yeast colonization differed significantly between patients with or without diabetes unless glycosuria was present, ${ }^{28}$ and a higher yeast carriage rate was scen in patients with poorly controlled diabetes in comparison with those patients with good diabetic control. ${ }^{29}$ In older women, vaginitis was increased significantly in those with insulin- 
dependent diabetes mellitus when compared with either healthy patients or diabetics not requiring insulin. ${ }^{21}$ It may be that our study did not demonstrate a difference in yeast carriage between diabetic and non-diabetic patients because glucose intolerance was less marked than that noted in previous studies.

In summary, yeast colonization was common among patients in the LTCF, with $84 \%$ of those patients who remained in the facility for 3 or more months colonized at some time during their stay. It is likely that colonization with yeasts is a prerequisite for infection, but the presence of other factors is probably also necessary for infection to develop. Colonization with yeasts in residents of a LTCF may not become clinically important until the patient is transferred to an acute-care hospital, where additional risk factors such as broad-spectrum antibiotic therapy and intravenous catheterization culminate in the development of serious yeast infection. In those LTCFs that have upgraded their services, e.g., those administering intravenous drugs and caring for patients receiving hemodialysis and those with chronic intravenous ports and catheters, serious Candida infections, including candidemia, may become a significant problem.

\section{ACKNOWLEDGMENT}

This study was supported by the Veterans Affairs Health Services Research and Development Service.

\section{REFERENCES}

1. Banerjee SN, Emori TG, Culver DH ct al. Secular trends in nosocomial primary bloodstream infections in the United States, 1980-1989. Am J Med 1991;91:86S-89S.

2. Jarvis WR, Martone WJ. Predominant pathogens in hospital infections. J Antimicrob Chemother 1991;28:15-19.

3. Voss A, Hollis RJ, Pfaller MA et al. Investigation of the sequence of colonization and candidemia in nonneutropenic patients. J Clin Microbiol 1994;32:975-980.

4. Reagan DR, Pfaller MA, Hollis RJ, Wenzel RP. Characterization of the sequence of colonization and nosocomial candidemia using DNA fingerprinting and a DNA probe. J Clin Microbiol 1990;28:2733-2738.

5. Wey SB, Mori M, Pfaller MA et al. Risk factors for hospital-acquired candidemia. Arch Intern Med 1989;149:2349-2353.

6. Bross J, Talbot GH, Maislin G, Hurwitz S. Risk factors for nosocomial candidenia: A case-control study in adults without leukemia. Am J Med $1989 ; 87: 614-620$.

7. Richet HM, Andremont A, Tancrede $\mathrm{C}$ et al. Risk factors for candidemia in patients with acute lymphocytic leukemia. Rev Infect Dis 1991;13:211-215.

8. Weems JJ, Chamberland ME, Ward J et al. Candida parapsilosis fungemia associated with parcnteral nutrition and contaminated blood pressure transducers. J Clin Microbiol 1987;25:1029-1032.

9. Isemberg I-ID, Tucci V, Cintron F et al. Single-source outbreak of Candida tropicalis complicating coronary bypass surgery. J Clin Microbiol $1989 ; 27: 2426-2428$.

10. Pertowski CA, Baron RC, Lasker BA et al. Nosocomial outbreak of Candida albicans sternal wound infections following cardiac surgery traced to a scrub nurse. J Infect Dis 1995;172:817-822.
11. Burnie JP, Odds FC, Lee W et al. Outbreak of systemic Candida albicans in intensive care unit caused by cross infection. Br Med J 1985;290:746-748.

12. Betremicux $P$, Chevrier $S, Q$ uindos $G$ et al. Use of DNA fingerprinting and biotyping methods to study a Candida albicans outbreak in a neonatal intensive carc unit. Pediatr Infect Dis J 1994;13:899-905.

13. Jackson MM, Fierer J, Barrett-Connor $E$ et al. Intensive surveillance for infections in a three-year study of nursing home patients. Am J Epidemiol $1992 ; 135: 685-696$.

14. Bradley SF, Terpenning MS, Ramsay MA et al. Methicillin-resistant Staphylococcus aurcus.colonization and infection in a long-term care facility. Ann Intern Med 1991;115:417-422.

15. Bonilla $\mathrm{HIF}$, Zervos MA, Lyons $\mathrm{MJ}$ et al. Colonization with vancomycinresistant Enterococcus faccium: Comparison of a long-term-care unit with an acute-care hospital. Infect Control Hosp Epideniol 1997;18:333-339.

16. Terpenning MS, Bradley SF, Wan JY et al. Colonization and infection with antibiotic-resistant bacteria in a long-term carc facility. J Am Geriatr Soc 1994:42:1062-1069.

17. Kauffman CA, Terpenning MS, He X et al. Attempts to eradicate methicillinresistant Staphylococcus aureus from a long-term care facility with the use of mupirocin ointment. Am J Med 1993;94:371-378.

18. Chenoweth $\mathrm{CE}$, Bradley SF, Terpenning MS ct al. Colonization and transmission of high-level gentamicin-resistant enterococci in a long-term care facility. Infect Control Hosp Epidemiol 1994;15:703-709.

19. Garner JS, Jarvis WR, Emori TG et al. CDC definitions for nosocomial infections, 1988. Am J Infect Control 1988;16:128-140.

20. SAS Institute Inc., SAS/STAT User's Guide, Version 6, 4th Ed. Cary, NC: SAS Institute, Inc., 1989.

21. Magaziner J, Tenney JI-I, DeForge B et al. Prevalence and characteristics of nursing home-acquired infections in the aged. J Am Geriatr Soc 1991;39:1071-1078.

22. Wilkieson C, Samaranayake LP, MacFarlane TW et al. Oral candidosis in the elderly in long term hospital care. J Oral Pathol Med 1991;20:13-16.

23. Jacobs LG, Skidmorc EA, Frecman K et al. Oral fluconazole compared with bladder irrigation with amphotericin $B$ for treatment of fungal urinary tract infections in elderly patients. Clin Infect Dis 1996;22:30-35.

24. Jacobs LG. Fungal urinary tract infections in the elderly. Drugs Aging $1996 ; 8: 89-96$.

25. Warren JW. The catheter and urinary tract infection. Med Clin North Am 1991;75:481-493.

26. Terpenning MS, Allada R, Kauffman CA. Intermittent urethral catheterization in the elderly. J Am Geriatr Soc 1989;37:411-416.

27. Sangeorzan JA, Bradley SF, He X ct al. Epidemiology of oral candidiasis in HIV-infected patients: Colonization, infection, treatment and emergence of fluconazole resistance. Am J Med 1994;97:339-346.

28. Mehnert B, Mehnert H. Yeasts in urine and saliva of diabetic and nondiabetic patients. Diabetes 1958;7:293-297.

29. Odds FC, Evans EGV, Taylor MAR, Wales JK. Prevalence of pathogenic ycasts and humoral antibodies to Candida in diabetic patients. J Clin Pathol 1978;31:840-844.

30. Marks MI, Marks S, Brazeau M. Yeast colonization in hospitalized and nonhospitalized children. J Pediatr 1975;87:524-527.

31. Contreras I, Ponton J, Quindos G. Prevalence of Candida parapsilosis in the oral cavities of infants in Spain. Clin Infect Dis 1994;18:480-481.

32. Hunter PR, Harrison GAJ, Fraser CAM. Cross-infection and diversity of Candida albicans strain carriage in patients and nursing staff on an intensive care unit. J Med Vet Mycol 1990;28:317-325.

33. Kleinegger CL, Lockhart SR, Vargas K, Soll DR. Frequency, intensity, species and strains of oral Candida vary as a function of host age. J Clin Microbiol 1996;34:2246-2254.

34. Stenderup A, Thomsen Pedersen G. Ycasts of human origin. Acta Pathol Microbiol Scand 1962;54:462-472.

35. MacKenzie DWR. Yeasts from human sources. Sabouraudia 1961;1:8-15. 